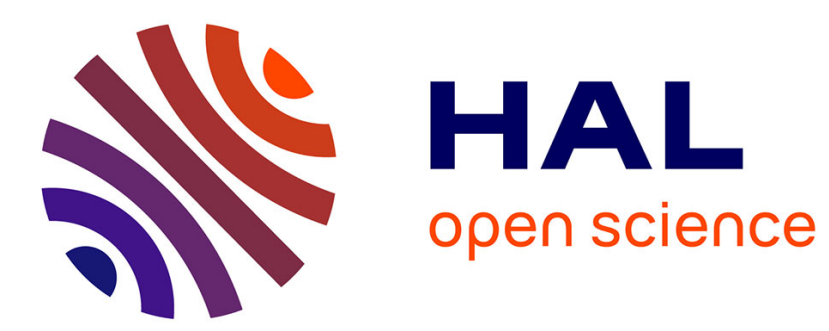

\title{
Approche des groupes culturels et de leurs interactions entre Rhin et Rhône à la transition Bronze-Fer \\ Laurie Tremblay-Cormier
}

\section{To cite this version:}

Laurie Tremblay-Cormier. Approche des groupes culturels et de leurs interactions entre Rhin et Rhône à la transition Bronze-Fer. Archimède: archéologie et histoire ancienne, 2014, 1, pp.217-227. halshs-01586672

\section{HAL Id: halshs-01586672 \\ https://shs.hal.science/halshs-01586672}

Submitted on 13 Sep 2017

HAL is a multi-disciplinary open access archive for the deposit and dissemination of scientific research documents, whether they are published or not. The documents may come from teaching and research institutions in France or abroad, or from public or private research centers.
L'archive ouverte pluridisciplinaire HAL, est destinée au dépôt et à la diffusion de documents scientifiques de niveau recherche, publiés ou non, émanant des établissements d'enseignement et de recherche français ou étrangers, des laboratoires publics ou privés. 


\title{
APPROCHE DES GROUPES CULTURELS ET DE LEURS INTERACTIONS ENTRE RHIN ET RHÔNE À LA TRANSITION BRONZE-FER
}

\author{
Laurie TREMBLAY CORMIER \\ Docteur, \\ UMR 6298-ARTeHIS, \\ Université de Bourgogne \\ laurietcormier@gmail.com
}

\section{RÉSUMÉ}

Les groupes culturels et leurs interactions découlent de dynamiques complexes, délicates à appréhender en Protohistoire en raison d'un grand nombre d'inconnues. Il demeure toutefois possible de les étudier en s'inspirant des modèles déjà établis, afin de mettre en place une approche tenant compte des divers types d'informations fournies par les vestiges archéologiques. L'analyse présentée ici est tirée d'une thèse de doctorat soutenue en avril 2013, touchant les groupes culturels et leurs dynamiques d'échanges dans une zone allant de la confluence SaôneRhône à l'embouchure de la Moselle, du Xe au viII ${ }^{\mathrm{e}} \mathrm{s}$. av. J.-C. Elle s'attache tout particulièrement au mobilier métallique qui, grâce à la prise en compte de la typologie et des modes de dépôt, servira d'indicateur culturel. L'analyse factorielle des correspondances, sa confrontation avec l'espace géographique et avec d'autres objets d'étude, permet d'identifier une série de groupes culturels pour l'extrême fin de l'âge du Bronze et les débuts du premier âge du Fer. Les interactions sont, pour leur part, approchées par le biais de

Mots-CLÉs

groupes culturels, mobilier métallique, interactions,

statistiques,

SIG.
Cultural groups and their interactions follow from complex dynamics, delicate to grasp due to many unknown factors. However, it is still possible to define an approach to this problem with the use of elements from pre-established models, taking in consideration the different types of information offered by archaeological remains. The present analysis is part of a doctorat defended in April 2013 about cultural groups and exchanges dynamics, in a study area going from the Saône-Rhône confluence to the Mosel's mouth, from the $10^{\text {th }}$ to the $8^{\text {th }}$ centuries BC. More particularly, this work used metalwork types and deposit context as cultural indicators. The controntation of the results from the factorial analysis with geographical space permits the characterization of series of cultural groups, at the very end of Bronze Age and the beginning of the Early Iron Age. In parallel, the interactions between these groups are analyzed through the «exchance flow» study, a method directly inspired by geographical sciences.
Keywords cultural groups, metalwork interactions, statistics, GIS. 


\section{INTRODUCTION}

Une définition fréquemment rencontrée des cultures, en sciences sociales, est celle d'entités complexes. Un groupe culturel est en effet polythétique [1], puisque composé d'un éventail de caractéristiques matérielles et immatérielles : coutumes, langues, croyances, mode de vie, lois, culture matérielle, savoirs scientifique et technique... Du point de vue de l'observateur extérieur, c'est l'ensemble de ces éléments qui permet de distinguer un groupe humain d'un autre [2], de créer des regroupements parmi les Hommes en fonction d'un partage de caractéristiques culturelles communes. Qu'en est-il pour la Protohistoire européenne ?

Avec des vestiges matériels soumis aux aléas de la taphonomie, des connaissances dépendantes de l'activité archéologique et de l'absence de sources écrites, le protohistorien est un observateur partiellement aveugle. Il ne voit, en effet, que l'artefact et les structures qui lui sont parvenus ; le reste est proposé, reconstitué, grâce à l'interprétation des vestiges. Ces indices se divisent entre les informations intrinsèques aux vestiges, directement perceptibles sur l'objet, et les informations extrinsèques, rattachables à son environnement [3]. Ce sont ces dernières qui, de par leur interprétation, permettent de reconstituer la part d'immatériel des cultures.

Au gré des études, de nombreux modèles d'interprétation ont été proposés pour la reconstitution des groupes culturels protohistoriques, s'inspirant de la linguistique, de la géographie, de l'ethnologie et même des mathématiques. À travers ce foisonnement de propositions, un modèle a été retenu ; celui-ci approche la question à l'aide du mobilier métallique, dans une aire d'étude sise entre le Rhin et le Rhône, du xe au viII ${ }^{\mathrm{e}}$ s. av. J.-C. Grâce à ce même mobilier métallique, une modélisation des réseaux d'interaction entre ces groupes culturels a également été réalisée.

\section{APPROCHE DES GROUPES CULTURELS}

\section{CHOIX DES INDICATEURS CULTURELS}

Deux aspects du mobilier métallique ont été retenus comme marqueurs culturels : les formes du mobilier et les pratiques de dépôt ; ils seront désignés, tout au long du travail, en tant qu'« indicateurs ».
Les formes du mobilier sont un indicateur relevant de la typologie, à la base de l'étude archéologique dans la détermination des systèmes de vestiges matériels [4]. On ne se limitera pas ici à un seul type d'objet : l'étude sera plutôt étendue à tous les types montrant un intérêt dans la recherche des groupes culturels.

Afin d'élargir le spectre des informations utilisées dans la recherche des groupes culturels, le mode de dépôt de l'objet a également été retenu comme indicateur. Cette information extrinsèque à l'objet est fournie par son contexte archéologique, qui correspond à sa dernière utilisation. Les circonstances entourant l'abandon définitif de l'objet peuvent ainsi être liées à une simple mise au rebut, au dépôt funéraire ou votif, à une thésaurisation... Cette variation repose sur l'existence de comportements humains différents, pouvant être considérés à juste titre comme des marqueurs culturels. On ne prendra ici en compte que les modes de dépôt dits « volontaires », où l'objet est posé dans une structure suivant un geste délibéré, à des fins relevant du domaine de l'abstraction. Cela exclut donc le mobilier domestique, pouvant résulter d'une perte lors de l'abandon de l'habitat ou ayant été volontairement laissé sur place, ce dernier cas s'apparentant alors à une mise au rebut [5]. Les indicateurs liés au dépôt volontaire dans l'aire et la période d'étude retenus se déclineront donc entre la tombe, le dépôt au sens d'un ensemble d'objets enfouis simultanément, les objets déposés en milieu humide et, dans certaines proportions, l'objet isolé - en milieu terrestre [6].

La distinction entre ces deux types d'indicateurs culturels a été posée très tôt dans les travaux de K.-H. JacobFriesen [7] sur le groupe de Lausitz, une approche inspirée des méthodes employées en ethnologie et qui est reprise dans les travaux de H.-J. Eggers. La distribution des types d'objets (Formenkreise) y est croisée aux manifestations culturelles (Kulturkreise), ces dernières pouvant être reliées aux pratiques de dépôt volontaire.

Parallèlement, I'optique géographique de l'approche de H.-J. Eggers [8] a été retenue pour l'élaboration du modèle. En effet, bien que le mobilier métallique soit au centre de l'étude, c'est l'espace qui est avant tout caractérisé pour devenir le véritable objet d'analyse. La carte de répartition des indicateurs culturels dépasse ainsi son simple rôle d'illustration pour devenir un outil de la réflexion; le croisement des répartitions, et non leur interprétation isolée, sert alors à l'interprétation culturelle des vestiges.

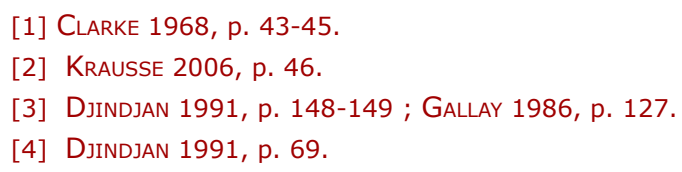

\author{
[5] EgGers 1959, p. 266. \\ [6] BRadley 1990, p. 4-14. \\ [7] JACob-Friesen 1928. \\ [8] Eggers 1950.
}




\section{MÉTHODE D'ANALYSE}

La méthode d'analyse s'échelonne sur trois niveaux emboîtés. Tout d'abord, une première étape consiste en la recherche des regroupements statistiques des indicateurs culturels présentés précédemment - les types et les modes de dépôt des objets - qui composent les «faciès ». En second lieu, la localisation de ces groupes statistiques dans l'espace donne corps aux « entités ». Finalement, celles-ci sont confrontées aux travaux antérieurs traitant d'autres objets d'étude, notamment le mobilier céramique et le costume funéraire, afin de proposer une série de groupes culturels (fig. 1).

Ces analyses ont pu être réalisées grâce à la mise en place d'une base de données relationnelle, fonctionnant selon une hiérarchie simple à trois niveaux : le site, la structure et l'objet. À l'origine créée sur Microsoft Access,

Figure 1 : niveaux d'analyse, depuis l'étude des indicateurs à la proposition de groupes culturels.

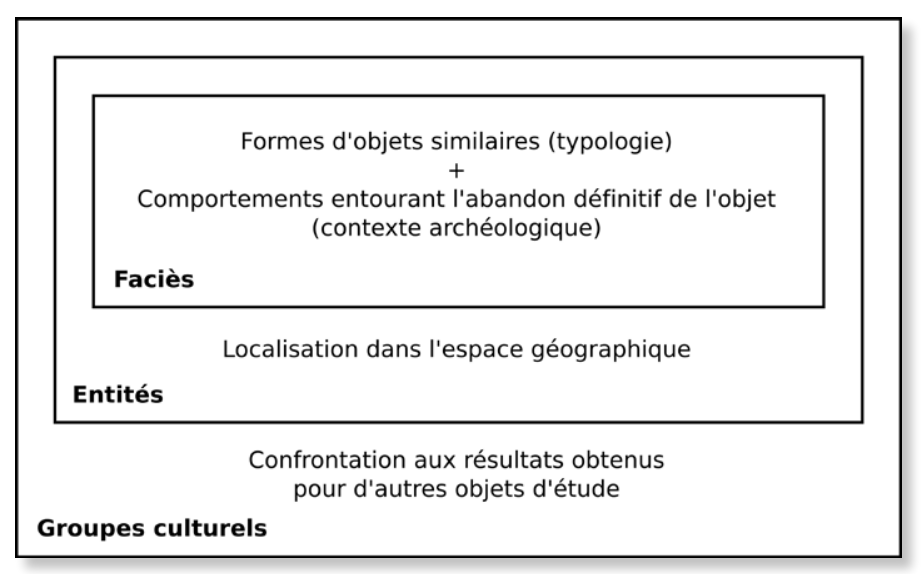

cette base a ensuite été transformée en format SQlite et SpatiaLite afin de faciliter son intégration au Système d'Information Géographique (SIG).

La première étape, soit la mise en évidence des faciès, a été réalisée à l'aide des Analyses Factorielles des Correspondances (AFC). En archéologie, ce type d'analyse est fréquemment employé dans le but de caractériser des assemblages d'ensembles clos comme les sépultures, les fosses d'habitats et les dépôts [9]. Cette méthode multivariée est dite « descriptive », puisqu'elle permet de synthétiser la distribution de variables selon leurs relations de dépendance, mettant en évidence des évolutions chronologiques (sériation) ou des regroupements selon des phénomènes sociaux, culturels ou géographiques (partition) dans l'agencement des données [10]. Ce dernier aspect des AFC est particulièrement intéressant pour la problématique, puisqu'il correspond précisément au but recherché, c'est-à-dire des regroupements d'indicateurs liés à des phénomènes culturels.

Toutefois, cette méthode est adaptée à l'analyse d'ensembles, ce qui exclut les objets isolés; de ce fait, il a été nécessaire d'adapter les AFC à l'analyse de l'espace géographique. Pour ce faire, les ensembles clos ont été remplacés par des unités géographiques, correspondant à une portion délimitée de l'espace et comprenant leur lot de vestiges à caractériser. L'analyse a donc été réalisée en cinq étapes successives (fig. 2 ) :

[9] DJINDJIAN 1991, p. 148.

[10] Bouroche \& SAPORTA 1980, p. 83-85; MAdSen 2007, p. 1-2.

Figure 2 : schéma de la méthode d'analyse employée.

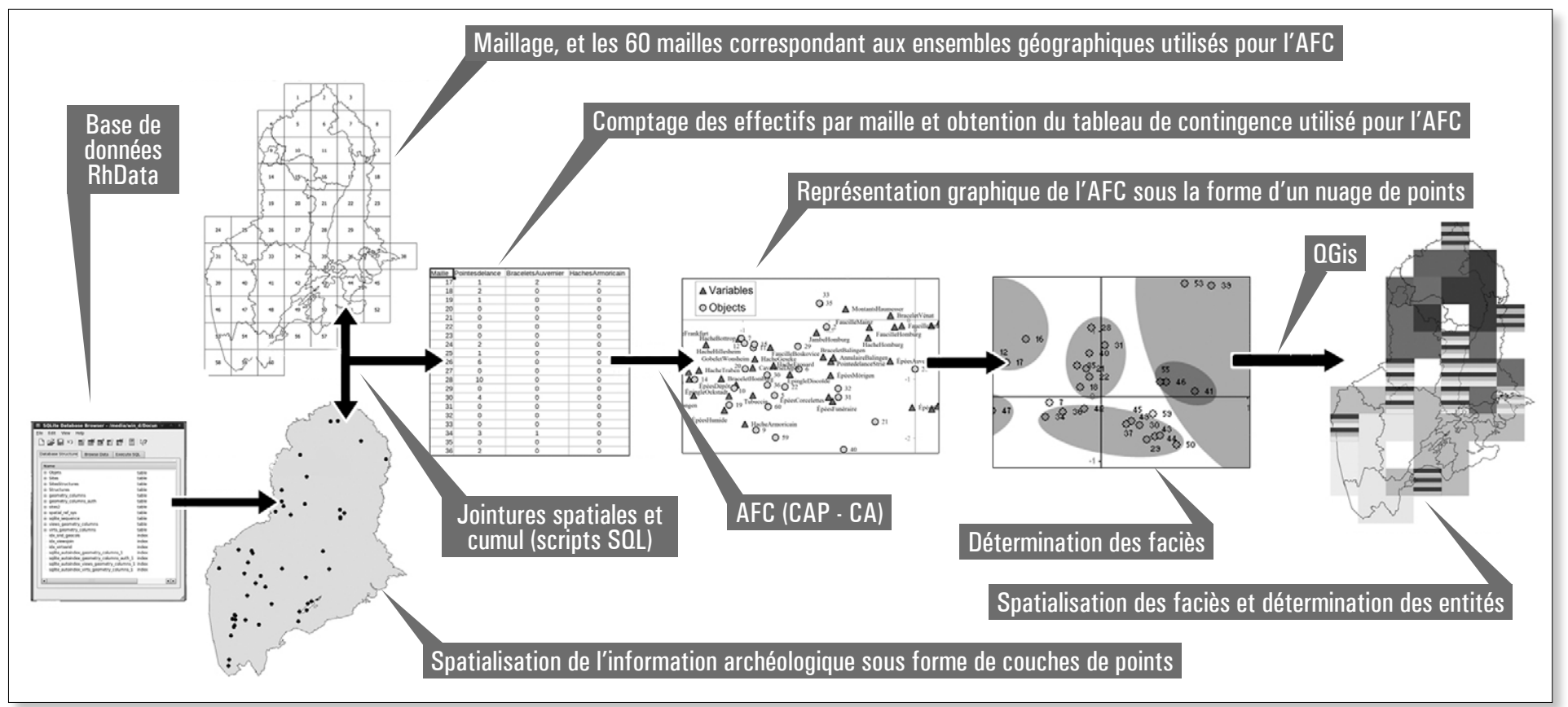


1. L'aire d'étude a tout d'abord été découpée par une grille de 60 mailles de $56 \mathrm{~km}$ de côté, réalisée à l'aide de I'outil Grille vecteur de QGis (version 1.7.1). Chacune de ces mailles joue le rôle d'une unité géographique, qui sera analysée dans I'AFC à la manière d'un ensemble clos.

À I'origine, la taille des mailles avait été fixée en employant une formule calculant le maillage optimal en fonction de la densité des vestiges : $\sqrt{ }($ Aire Zone / Quantité Points). Le résultat correspondait à des mailles de 14 km de côté, ce qui était trop petit pour créer des regroupements statistiquement valables pour I'AFC. La taille des mailles a donc été augmentée de manière empirique, jusqu'à l'obtention de mailles d'une emprise satisfaisante.

2. Parallèlement, les données archéologiques ont été projetées sous la forme de points directement depuis la base de données, grâce à I'extension QSpatiaLite. Cette manipulation a l'avantage de permettre la réalisation de requêtes spatiales directement sur les données [11]. Chaque indicateur a ainsi pu être spatialisé en tant que couche de points individuelle dans le SIG réalisé avec QGIS 1.7.1.

3. Le croisement des données archéologiques et du maillage a ensuite été réalisé grâce à une série de scripts SQL2 lancés dans une console Unix. Grâce à une jointure spatiale entre le maillage et chaque couche de points, un premier comptage de chaque indicateur par maille a pu être réalisé. Ces informations ont ensuite été regroupées dans une couche cumulative résumant la quantité de chaque indicateur par maille.

La table attributaire de cette dernière couche est composée des mailles, en rangées, et des indicateurs, en colonnes. Les données correspondent ainsi à la quantité respective de chaque indicateur par maille.

4. Cette table attributaire cumulative joue également le rôle de tableau de contingence, utilisé dans I'AFC. L'analyse a été réalisée à l'aide de I'extension CA-PCA 2.11 [12] pour Microsoft Excel 2007. Afin d'éliminer les valeurs extrêmes, les mailles possédant moins de trois indicateurs ont été retirées, de même que les indicateurs présents en moins de quatre exemplaires, ceci afin d'exclure les unités et variables statistiquement non valables.

La représentation graphique de cette analyse, à partir du calcul du $x 2$, est un nuage de points multidimensionnel possédant autant de plans factoriels qu'il y a de critères à analyser [13]. Les plans factoriels ont été ici limités à trois, afin de faciliter la lecture des résultats. L'interprétation des résultats est finalement réalisée en fonction de la proximité entre les points sur le graphique, et de leur positionnement par rapport aux autres critères [14]. On cherchera en effet à expliquer la proximité des points représentant les unités géographiques par la détermination d'assemblages d'indicateurs similaires, correspondant aux faciès recherchés.

5. La confrontation des regroupements statistiques d'indicateurs avec la représentation cartographique permet enfin de valider ou d'invalider ces faciès comme des entités, et de proposer une première modélisation. La comparaison des entités retenues avec les travaux antérieurs permet enfin de proposer des séries de groupes culturels, ici pour les périodes du Hallstatt B2/B3 ( $\mathrm{Ha}$ B2/B3) et du Hallstatt C ( $\mathrm{Ha} \mathrm{C})$.

\section{RÉSULTATS}

\section{Ha B2/B3}

Les groupes culturels obtenus par la définition des faciès, puis des entités, et finalement par leur confrontation avec d'autres indicateurs, montrent deux tableaux en continuité pour le $\mathrm{Ha}$ B2/B3 et le Ha C. Cependant, des variations non négligeables trahissent d'importants changements attribuables à une réorganisation culturelle qui se poursuivra tout au long du premier âge du Fer.

Le $\mathrm{Ha}$ B2/B3 comprend 143 indicateurs, contre 54 au Ha C. Après exclusion des indicateurs présents à moins de quatre individus et des mailles comprenant trois indicateurs ou moins, I'AFC concerne respectivement 5218 et 1166 objets, ainsi que 47 et 40 mailles. Cet important écart s'explique par la baisse générale du nombre d'objets déposés dans les tombes, I'abandon des sites lacustres ainsi que la quasi-disparition des dépôts au $\mathrm{Ha}$ C. Fort heureusement, cette baisse du nombre d'objets disponibles pour l'analyse a pu être compensée par la prise en compte de travaux sur le mobilier céramique.

Les groupes du Ha B2/B3 se divisent entre trois grandes régions : le sud-ouest de l'Allemagne, l'ouest de la Suisse et le nord-est de la France (fig. 3). Un premier groupe est visible aux abords de la confluence Rhin-Main et s'étend à la Sarre, au Palatinat et au Rhin moyen (carte fig. 3,1$)$. Il se caractérise par de nombreux dépôts au taux de fragmentation élevé, comportant principalement des armes, des éléments de char, des haches et des outils [15]. L'étude des formes céramiques [16] montre I'existence d'un second groupe, dans le sud de la Hesse (carte fig. 3, 2), partageant ce même faciès métallique.

[11] FURIERI 2012.

[12] MADSEN 2012.

[13] Lebart, Morineau \& Fénélon 1982, p. 308 ; Millet 2008, p. 194.

[14] Bouroche \& SaPORTA 1980, p. 98 ; FÉnelon 1981, p. 151-152; Lebart, Morineau \& FÉnelon 1982, p. 320.

[15] HANSEN 1991, p. 157-158.

[16] Herrmann 1966, p. 34. 
Figure 3 : groupes culturels proposés au Ha B2/B3 et principaux indicateurs culturels retenus au sein du mobilier métallique.

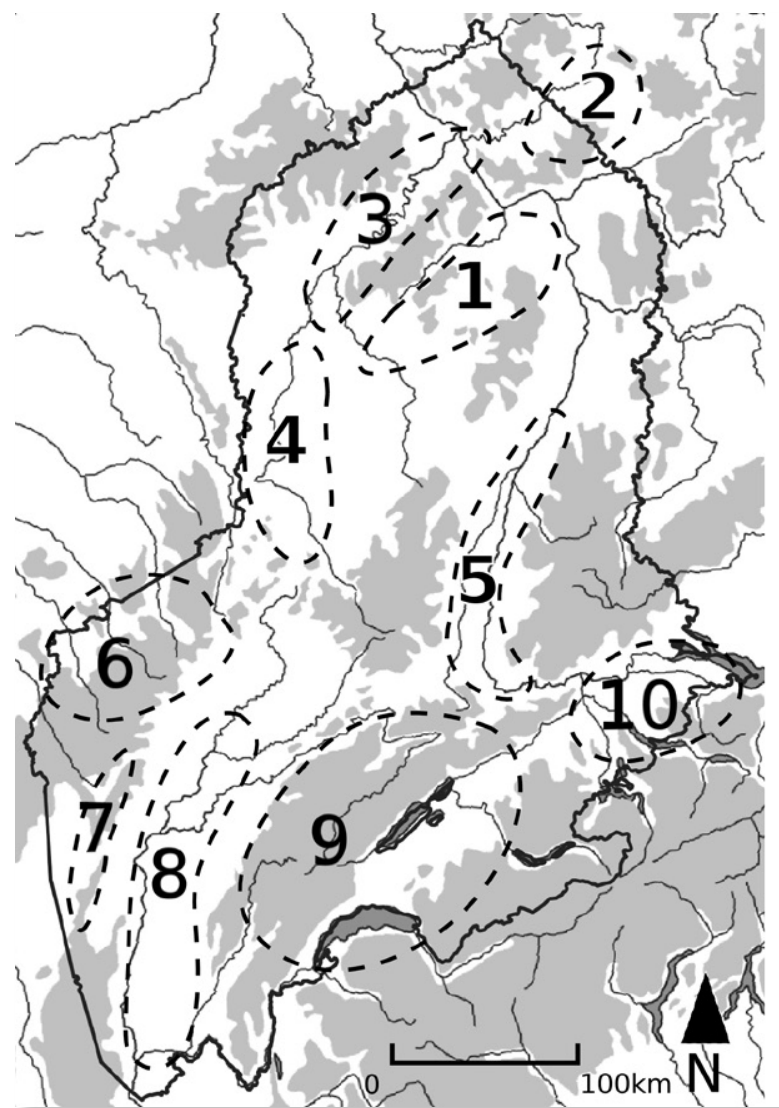

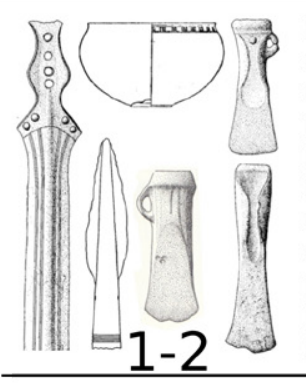

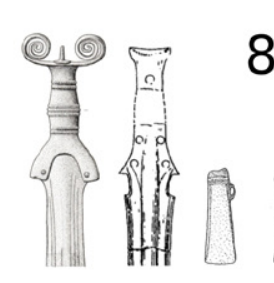

8

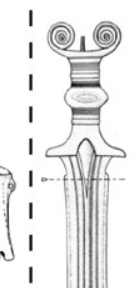

$-\frac{1}{1}$

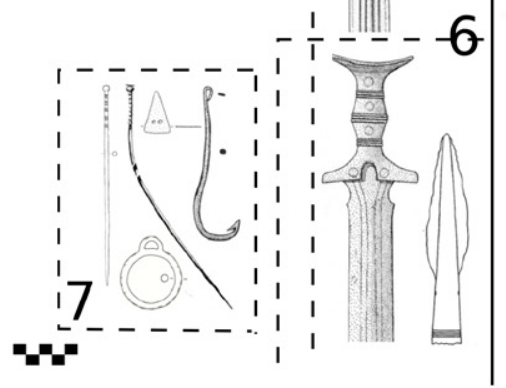

(1)
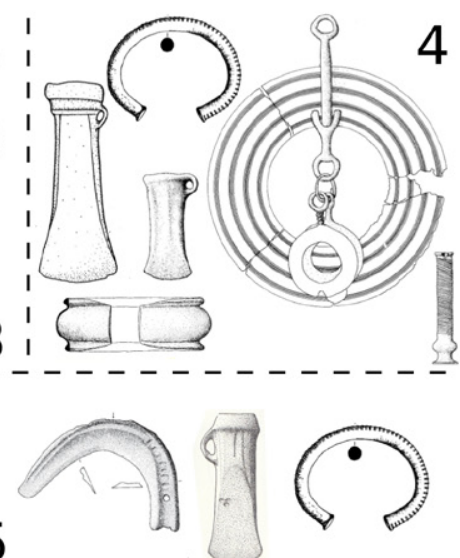

9-

10
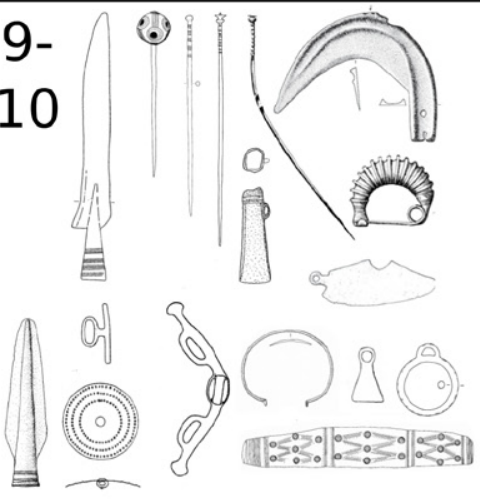

1-2: épée type Großauheim; gobelet type Wonsheim; hache à ailerons figurés; pointe de lance striée ; hache type Bottrop; hache type Hillesheim.

3 : épingle à tête discoïde; faucille type Auvernier; hache type Frouard; hache type Geseke-Biblis; parure annulaire type Homburg.

4 : parure annulaire type Homburg; hache type Traben-Trabach ; hache type de l'Elbe moyen ; tintinabulum ; parure annulaire type Wallerfangen; tubuccin.

5 : faucille type Boskovice; hache type Bottrop ; parure annulaire type Homburg.

6 : épée type Corcelettes; épée type Mörigen; pointe de lance striée.

Les études antérieures sur la vallée de la Moselle en font une zone relativement homogène en ce qui a trait aux décors céramiques, aux modes de dépôt et à la pratique de l'incinération sous tumulus [17]. Or, I'AFC fait une distinction entre un groupe de la Moselle inférieure (carte fig. 3, 3), allant du bassin de Neuwied au Luxembourg, et un second le long de la Moselle supérieure, englobant le bassin de Trèves et la Lorraine (carte fig. 3, 4). La cohésion entre la Lorraine et la Sarre est confirmée par l'assemblage des dépôts, la composition chimique du mobilier et des techniques de fabrication communes [18]. L'analyse factorielle montre

[17] Kimmig 1952, p. 159 ; Kolling 1968.

[18] VÉBER 2003.
7 : épingle à tête globulaire ; épingle à tête vasiforme variante 1 ; pointe de flèche type Le Bourget; hameçon; pendeloque annulaire.

8 : épée type Tachlovice; épée en langue de carpe; hache type armoricain; hache type Maure; épée type Corcelettes; épingle à tête globulaire ; épingle à tête vasiforme variante 1 ; pointe de flèche type Le Bourget; hameçon ; pendeloque annulaire.

9-10 : couteau à douille; épingle céphalaire ; épingle globulaire ; épingle à tête vasiforme variante 2 ; épingle à tête vasiforme variante 3 ; faucille type Herrnbaumgarten; fibule type Mörigen ; hache type armoricain ; rasoir type Auvernier; pointe de lance astragalée ; montant de mors type Hauterive ; phalère ; montant de mors type Wallerfangen; parure annulaire type Corcelettes; pendeloque triangulaire; pendeloque annulaire.

une préférence de ce groupe pour les haches de type Traben-Trachbach et de I'Elbe moyen, les parures annulaires de type Homburg et Wallerfangen, les tubuccins et les tintinnabula entiers ; pour sa part, le groupe de la Moselle inférieure est davantage tourné vers les haches de type Geseke-Biblis et Frouard, les épingles à tête discoïde et les faucilles de type Auvernier. Cette distinction demeure néanmoins mince et gagnerait à être confrontée à d'autres objets d'étude.

Un dernier groupe peut être rattaché au sud-ouest de I'Allemagne, celui du Rhin supérieur (carte fig. 3, 5). En raison de la faible quantité de mobilier métallique provenant de cette zone, ce groupe n'est que discrètement visible dans I'AFC grâce à une proportion plus forte des haches de type Bottrop, des bracelets de type 
Homburg et des faucilles de type Boskovice. Il se signale toutefois dans la production céramique par l'emploi du graphite et de la peinture rouge [19], avec de nettes affinités avec le nord de la Suisse [20].

Le quart nord-est de la France est représenté par du mobilier aux types exogènes, principalement lacustres ou atlantiques. Certains de ces objets peuvent toutefois avoir été produits dans le nord-est de la France en reproduisant des schémas « étrangers », et demeurent ainsi délicats à distinguer des véritables importations. Néanmoins, quelques cas témoignent clairement d'une métallurgie locale, comme les épées d'Uchizy et d'Ouroux-sur-Saône, dont la poignée imitant le type Mörigen a été coulée sur une lame en langue de carpe d'origine atlantique [21]. Les modes de dépôt du mobilier jouent toutefois un rôle important en tant que marqueurs culturels.

Sur les plateaux du nord de la Côte d'Or et du sud de la Haute-Marne, on distingue le groupe du ChâtillonnaisMontsaugeonnais (carte fig. 3,6). Celui-ci est visible dans I'AFC par une plus forte proportion d'épées à manche massif et de pointes de lance striées, de même que par sa pérennité dans les phases suivantes. Il se détache d'un groupe plus restreint sis en rive droite de la Saône, sur les côtes de Beaune et du Mâconnais (carte fig. 3, 7). Ce groupe est en lien avec les nécropoles de Verzé et des Chaumes d'Auvenay, où I'on retrouve une plus forte proportion d'épingles à tête globulaire et à tête vasiforme de type 1 , ainsi que des pendeloques annulaires. Ces parures sont partagées avec un groupe englobant I'est de la Bourgogne et la Franche-Comté (carte fig. 3, 8). Ce dernier se caractérise également par de nombreux dépôts, une forte présence de l'armement et des importations atlantiques, plus particulièrement de haches armoricaines et de type Maure, ainsi que d'épées en langue de carpe.

Finalement, la Suisse se divise en deux groupes au $\mathrm{Ha}$ B2/B3, malheureusement invisibles dans I'AFC. En effet, la surreprésentation du mobilier des stations lacustres de la région des Trois Lacs (carte fig. 3, 9 ; I'ensemble du mobilier, trop nombreux, n'est représenté que par les principaux types) tend à masquer le nord-est de la Suisse (carte fig. 3, 10). Ce dernier groupe est toutefois attesté par les formes et les décors de la céramique [22].
De fortes affinités réciproques dans le corpus céramique tendent également à masquer la visibilité de ces deux groupes, qui sera néanmoins plus marquée au Ha C [23].

\section{Ha C}

Au Ha C (fig. 4), l'aire d'étude se divise non plus en trois mais en quatre grandes régions. Dans le sud-ouest de I'Allemagne, le groupe Rhin-Main du Ha B2/B3 se divise entre la Sarre-Palatinat (carte fig. 4, 1) et la Hesse rhénane (carte fig. 4, 3), partageant un faciès métallique similaire. Tandis que le premier montre un passage rapide à l'inhumation, ainsi que des affinités avec le Rhin supérieur par un abondant dépôt de céramiques fréquemment décorées de graphite et de peinture rouge [24], le second se tourne vers la Lorraine et le sud de la Hesse, avec une plus forte représentation des rasoirs, des bracelets à œillets et décorés de nervures [25], caractéristiques de la fin du $\mathrm{Ha} \mathrm{C}$ et du Ha D1. Pour sa part, le sud de la Hesse (carte fig. 4, 2) demeure en continuité avec l'extrême fin du Bronze final et compose le groupe de Koberstadt, identifié depuis le début du $x x^{e}$ s. pour le Wetterau et le Main inférieur [26] ; il partage également des indicateurs similaires au sein du mobilier métallique.

La vallée de la Moselle n'est pas attestée par l'AFC au Ha $C$, en raison de l'absence de mobilier métallique déposé dans les tombes. Fort heureusement, les travaux antérieurs ont mis en évidence le groupe de Laufeld (carte fig. 4,4 ), en forte continuité avec le bassin de Neuwied au Ha B2/B3 et caractérisé par la pratique de l'incinération sous tumulus, la crémation du mobilier funéraire et un vaisselier complexe montrant, vers l'ouest, quelques influences de la culture de Haulzy [27].

Le groupe du Rhin supérieur (carte fig. 4, 5), qui présentait un problème similaire au groupe de Laufeld au Ha B2/B3, est désormais bien attesté grâce à plusieurs ensembles funéraires. Il partage, avec le nord-est de la Suisse, la présence de parures annulaires de type Berne, Schotz et Tschugg, de pointes de lance, de coupes métalliques à large bord, de couteaux, d'éléments de char et de harnachement, ainsi que de pincettes. Ces éléments attestent des affinités fortes avec le Wurtemberg et le sud de la Bavière [28], tout comme la céramique de type Alb-Hegau [29].
[19] Kimmig 1940 , p. $122 ; 1954$, p. $159-160$.

[20] Grimmer-DeHn 1991, p. 74-75.

[21] BONNAMOUR 1990, p. 39.

[22] VoGt $1930 ; 1942$, p. 205.

[23] Bolliger 2001, p. 35 ; Mäder 2001, p. 78.

[24] KoEPKE 1998, p. 64-67.
[25] Olivier 1993, p. 351 ; Reinhard 2003, p. 67-69.

[26] Dielmann 1952 ; Schumacher 1918 ; Kubach 1994, p. 235-236.

[27] DeHn 1936, p. 16 ; HaffNer 1973, p. 416 ; HaffNer 1976, p. 86 ; HoRnUNG 2008, p. 282 ; JoAChim 1968, p. 14 ; KRAUSE 1991, p. 49.

[28] ZÜRN 1987 ; KOSSACK 1959.

[29] Balzer 2009, p. 515 ; Koenig \& Adam 2005, p. 280 ; Maise 2001 ; Mentele, KuHnLe \& LASSERRE 2005. 


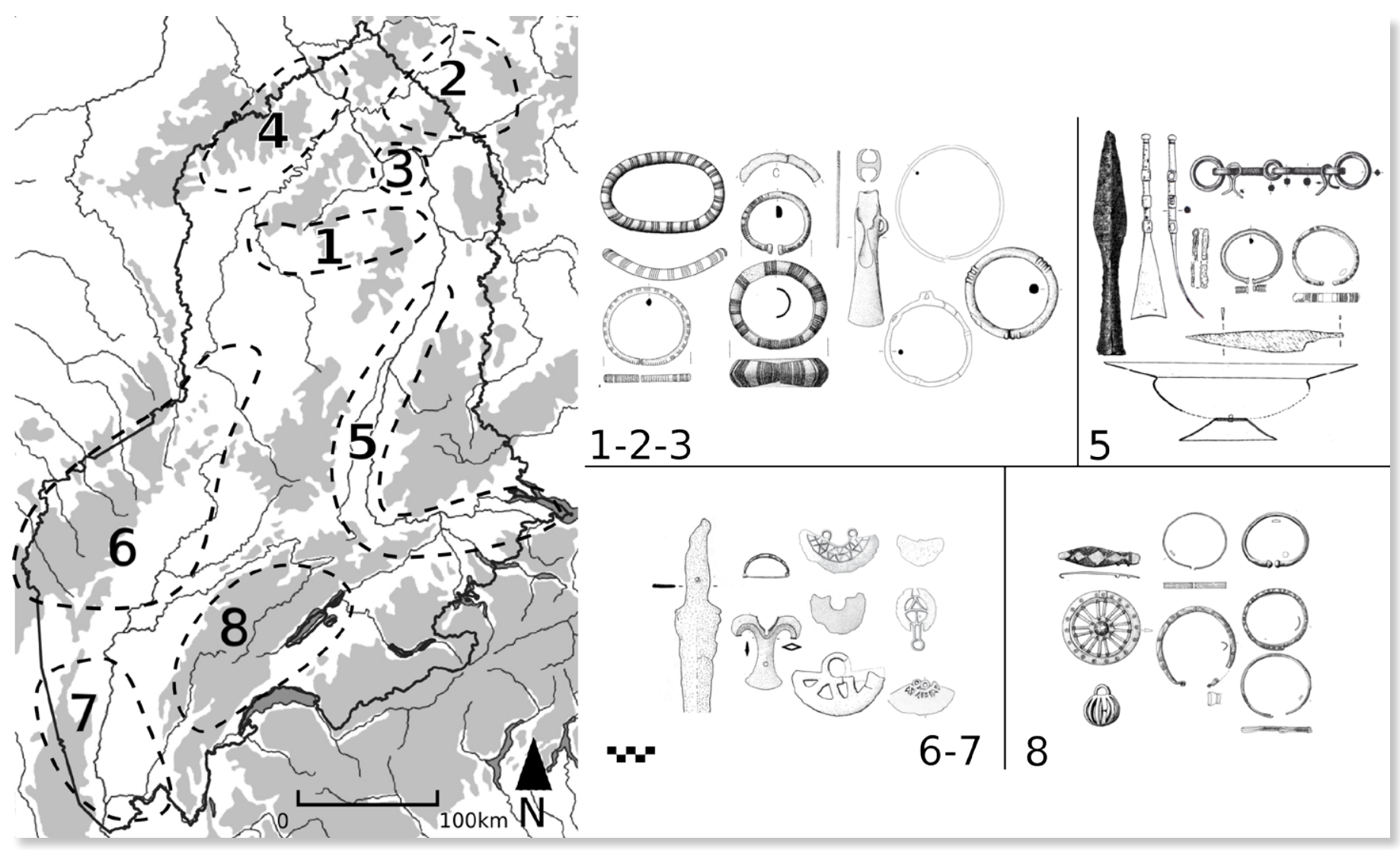

1-2-3: anneau de jambe coudé ; parure annulaire type 31 ; parure annulaire type Koberstadt; épingle torsadée; hache à ailerons subterminaux; torque de section circulaire; parure annulaire type 10 ; parure annulaire type Mittelgebirgszone ; parure annulaire à oeillets ; parure annulaire décorée de groupes de cannelures.

5 : pointe de lance; mors type Maxglan; mors type Frankfurt; pincette; parure annulaire type Tschugg variante; parure annulaire type Schötz; couteau ; coupe à large bord.

Dès le début du premier âge du Fer, le nord-est de la France se réorganise en deux groupes; toutefois, ce sont à nouveau les modes de dépôt du mobilier qui permettent d'effectuer cette distinction. Un premier groupe englobe une vaste zone allant du sud de la Lorraine à I'ouest de la Franche-Comté et au Châtillonnais (carte fig. 4,6$)$; il s'organise autour des tombes à épées, où se retrouvent fréquemment les bouterolles et rasoirs de type Clayeures, Dampierre, Magny, Mauvilly, Poiseul et Sundhoffen. Autour de la confluence Saône-Rhône (carte fig. 4, 7), la poursuite du dépôt d'objets en milieu humide porte à y voir un second groupe, une distinction également visible dans les productions céramiques [30].

Pour sa part, l'ouest de la Suisse (carte fig. 4, 8) demeure en continuité avec le Ha B2/B3, tant dans les formes céramiques que les formes de la parure annulaire. Paradoxalement, cette continuité de la culture matérielle
6-7 : épée en fer; fibule italique ; bouterolle type Beratzhausen; rasoir type Clayeures ; rasoir type Mauvilly-Sundhoffen ; rasoir type Dampierre ; rasoir type Minot ; rasoir type Magny-Lambert ; rasoir type Poiseul.

8 : agrafe de ceinture foliacée ; parure annulaire type Belp ; parure annulaire type La Béroche ; disque ; parure annulaire type Gorgier ; parure annulaire type Subingen; pendeloque-grelot; parure annulaire type Valangin.

s'accompagne d'une rupture dans les modes d'occupation du territoire, avec l'abandon des stations lacustres au profit de l'habitat ouvert dispersé, et de la disparition du dépôt de mobilier en milieu humide [31]. Ce groupe inclut le versant ouest du massif jurassien et demeure distinct du nord de la Suisse, une séparation visible dans les décors céramiques du canton de Fribourg, où la peinture rouge et le graphite sont absents [32]. Il se caractérise par de nombreuses parures comme des agrafes de ceinture, des disques, des grelots et une parure annulaire de type Belp, La Béroche, Gorgier, Subingen et Valangin, annonçant le Ha D1.

[30] Labeaune 2009, p. 190-191; Ramponi 2009, p. 147 ; Vital 1993, p. $147-149$.

[31] DUNning \& RYCHner 1994, p. 84-89.

[32] RufFieuX \& MAUVILly 2009, p. 505-506. 


\section{QUANTIFICATION DES} INTERACTIONS CULTURELLES

\section{LES INDICATEURS COMME TÉMOINS DES INTERACTIONS}

L'approche précédente a permis, en confrontant I'analyse statistique à l'espace géographique et à d'autres objets d'études, de proposer une série de groupes culturels entre Rhin et Rhône pour la transition Bronze-Fer. Certains groupes déjà pressentis auparavant grâce aux travaux antérieurs ont ainsi pu être confirmés d'une manière quantitative, tandis que d'autres ont été mis en évidence grâce à I'AFC.

L'analyse multivariée a également pointé le partage d'indicateurs entre certains groupes culturels, c'est-àdire la présence d'objets métalliques similaires dans des groupes différents. Cette observation fournit ainsi la première étape d'un nouvel aspect des sociétés protohistoriques, celui de leurs interactions, prises au sens des échanges entre les groupes composant l'aire d'étude.

Les indicateurs caractéristiques d'un groupe, qui sont retrouvés hors de ce dernier, trahissent en effet des phénomènes à l'origine des interactions culturelles. En l'occurrence, ici, le déplacement d'un objet métallique d'une région vers une autre peut être associé à deux dynamiques: la circulation de produits ou de savoirfaire, dans le cadre des échanges, ou la circulation du propriétaire de l'objet, associée aux déplacements d'individus et de populations [33].

\section{MÉTHODE D’ANALYSE}

La quantification des interactions permet de rendre compte de l'intensité de la relation liant deux groupes. Celle-ci a été réalisée par le calcul des « flux d'échanges », une méthode utilisée en géographie [34], employant le nombre d'indicateurs propres à une région retrouvés dans une autre pour l'obtention d'un indice de relation. Le calcul des flux d'échanges se faisant toujours d'une entité géographique $A$ vers une entité $B$, la relation calculée $n$ 'est pas bilatérale, mais témoigne des flux entrants et sortants de chacune.

Cette méthode débute par la réalisation d'un tableau d'échanges, recensant le nombre d'indicateurs d'une entité $A$ retrouvé dans toutes les autres entités de l'aire d'étude. Les flux entrants et sortants, c'est-à-dire la quantité d'indicateurs « émis » et « reçus » par chaque entité, est ensuite calculée et pondérée. Le résultat final est un indice de relation, témoignant de la force de l'interaction entre chaque couple d'entités de l'aire d'étude.

Cette méthode se heurte cependant à une limite majeure. En effet, force est de reconnaître que le lieu de fabrication de la majorité des objets échappe à l'archéologue, hormis dans quelques cas où des produits semi-finis, liés à la production d'un type précis, ont été identifiés. Dès lors, il devient délicat d'attribuer une région d'origine aux indicateurs ! Ce problème a été contourné en utilisant la région de concentration comme point d'origine des objets dont le lieu de fabrication exact reste inconnu.

De plus, certains groupes partagent un faciès métallique commun, à l'instar de la Sarre-Palatinat (fig. 4, 1) et de la Hesse rhénane (fig. 4, 3) au $\mathrm{Ha} C$, ce qui rend le calcul des flux d'échanges impossible. En effet, comment voir le déplacement d'indicateurs entre deux zones, si celles-ci sont identiques sur le plan du mobilier métallique ? Pour cette raison, une fusion a été opérée entre les groupes possédant le même assemblage d'indicateurs. L'analyse concerne alors des « régions » plutôt que des groupes culturels ; ce choix, bien que moins précis, offre ainsi des résultats plus pertinents.

Les interactions ont finalement été matérialisées sur un fond cartographique par des flèches, dont l'épaisseur varie selon l'indice de relation obtenu. La modélisation de cet indice offre, de cette manière, un résultat plus visuel (fig. 5 et 6) des interactions entre les groupes culturels pré-cités.

\section{RÉSULTATS}

\section{Ha B2/B3}

Les indicateurs du mobilier métallique de l'extrême fin du Bronze final sont principalement issus des dépôts et des sites lacustres suisses. Les indices de relation indiquent des interactions privilégiées entre les groupes de l'ouest de la Suisse (C) et du sud-ouest de l'Allemagne (A) (fig. 5). Les indicateurs suisses concernent les rasoirs à tranchant unilatéral, la parure annulaire (types Corcelettes, Mörigen), les faucilles (types Corcelettes, Herrnbaumgarten) et les mors (type Hauterive, Wallerfangen) ; le sud-ouest de l'Allemagne est, pour sa part, représenté par les tubuccins, les haches (types Frouard et Geseke-Biblis), les épingles à tête discoïde, les faucilles de type Boskovice et les parures annulaires de type Homburg.

Des interactions de moindre importance relient l'ouest de la Suisse (C) au nord-est de la France (B). À l'inverse, cette dernière région est celle montrant les interactions les plus faibles; cela est dû à la caractérisation de ses groupes par des indicateurs exogènes (productions atlantiques, lacustres ou du sud-ouest de l'Allemagne), rendant ainsi impossible le calcul des flux d'échanges sortants.

[33] Pierrevelcin 2012, p. 35-36

[34] Pumain \& Saint-Julien 2001, p. 14 


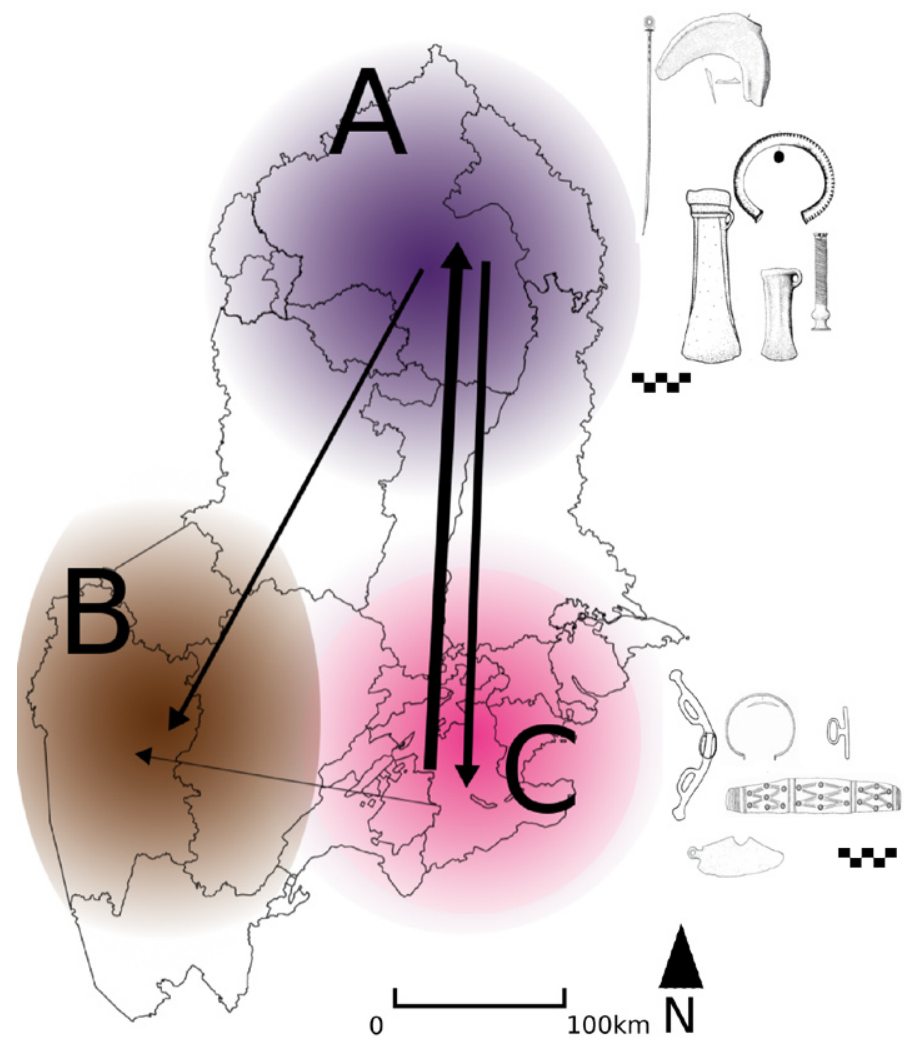

Figure 5 :

flux d'échanges calculés au $\mathrm{Ha}$ B2/B3 et résumé des indicateurs d'échange retenus.

A : épingle à tête discoïde ; faucille type Boskovice ; parure annulaire type Homburg; hache type Geseke-Biblis ; hache type Frouard ; tubuccin.

C : montant de mors type Wallerfangen ; parure annulaire type Corcelettes ; montant de mors type Hauterive ; rasoir type Auvernier.

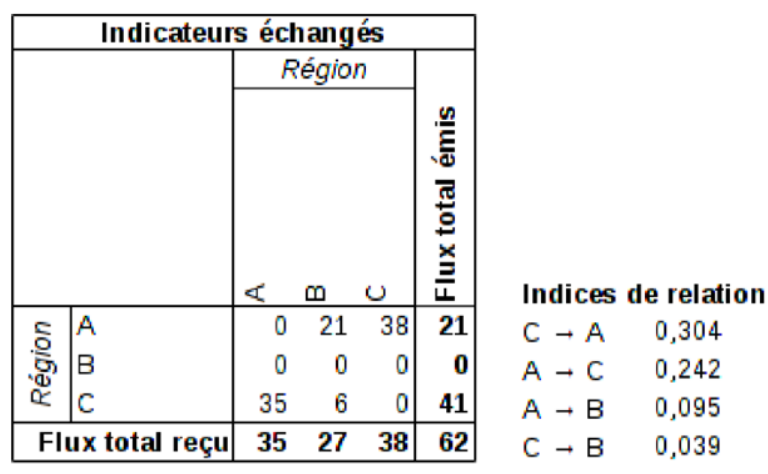

Figure 6 :

flux d'échanges calculés au Ha $\mathrm{C}$ et résumé des indicateurs d'échange retenus.

A : anneau de jambe coudé ; parure annulaire type Schötz variante.

B : parure annulaire type Tschugg variante ; parure annulaire type Schötz.

C : parure annulaire type Belp.

D : rasoir type Clayeures ; rasoir type Minot.

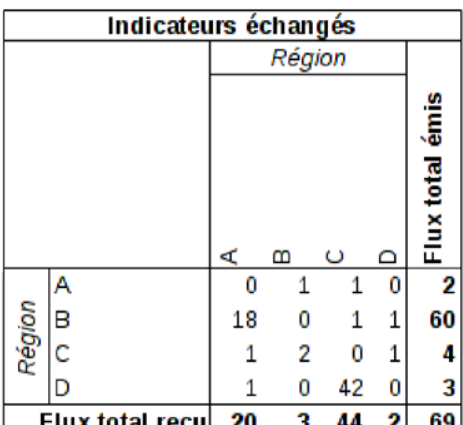

Indices de relation

$D \rightarrow C \quad 0,446$

$B \rightarrow C \quad 0,213$

$A \rightarrow C \quad 0,319$

$B \rightarrow$ A $\quad 0,097$

$\mathrm{D} \rightarrow \mathrm{A} \quad 0,087$

C $\rightarrow$ A 0,072

$\mathrm{C} \rightarrow \mathrm{B} \quad 0,022$

$\mathrm{A} \rightarrow \mathrm{B} \quad 0,022$

$B \rightarrow D \quad 0,010$

$C \rightarrow D \quad 0,007$

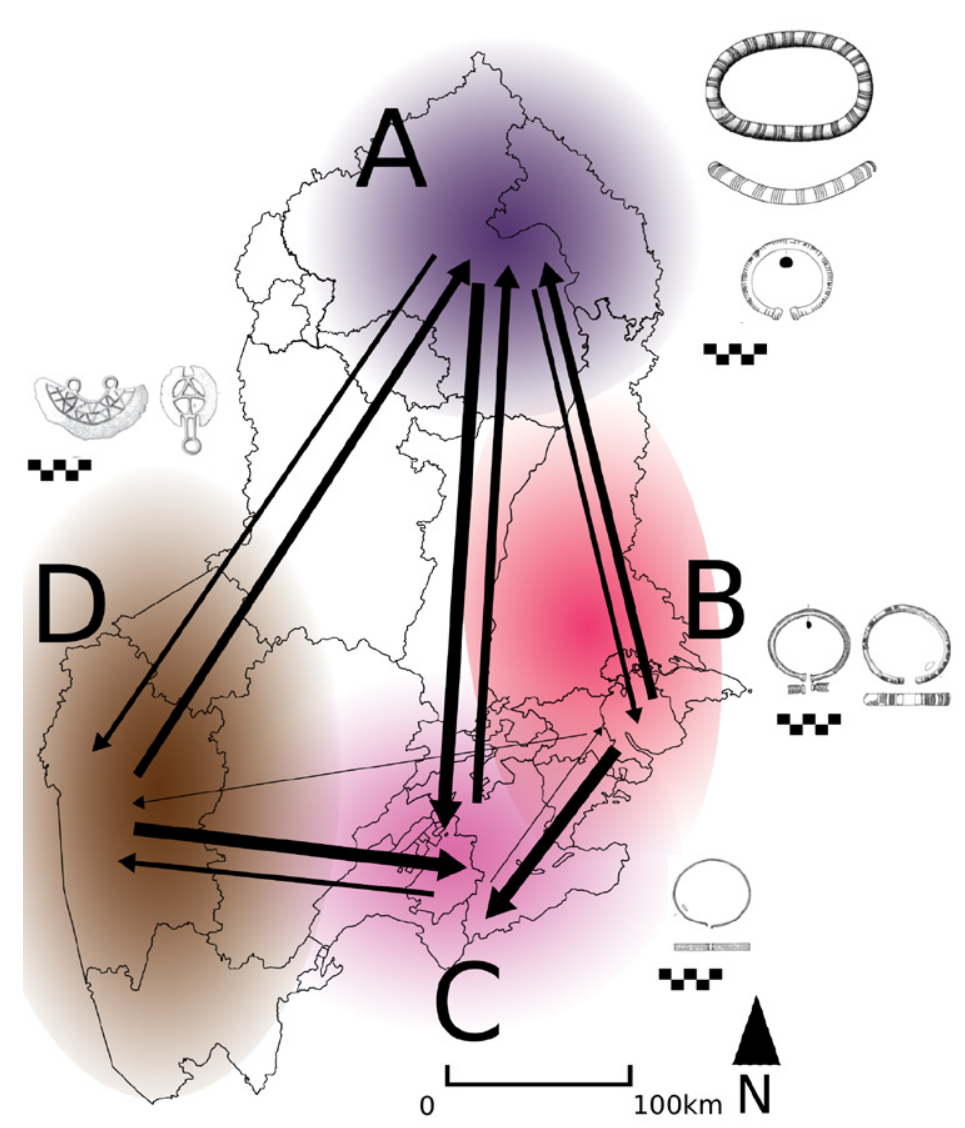




\section{Ha C}

Un schéma similaire demeure au Ha C malgré l'individualisation du Rhin supérieur (D) (fig. 6). Cette nouvelle région offre les flux sortants les plus forts, principalement matérialisés par des parures annulaires (types Berne, Schötz, Tschugg et variantes) ; paradoxalement, les relations vers le Rhin supérieur sont parmi les plus faibles, avec de rares indicateurs en provenance de Suisse (C) et du nord-est de la France (B).

Les interactions vers l'ouest de la Suisse (C) depuis le sud-ouest de l'Allemagne (A), de même que depuis le nord-est de la France (B), viennent au second rang. Elles sont respectivement représentées par de la parure annulaire (types Mittelgebirgszone, variante Schötz) et des rasoirs (types Clayeures, Minot). L'ouest de la Suisse (C) est, pour sa part, représenté par de la parure annulaire de type Belp.

La réduction du spectre des indicateurs est flagrante, celle-ci passant d'outils, de parures et d'éléments de harnachement, au Ha B2/B3, à des parures annulaires et des rasoirs. Ce phénomène s'explique par la forte diminution du nombre de dépôts et de sites lacustres au Ha $C$, période à laquelle la majorité des indicateurs est issue du contexte funéraire, où la parure annulaire et les instruments de toilette tiennent une place de choix dans les assemblages.

\section{CONCLUSION}

L'étude des groupes culturels et des interactions, par le biais du mobilier métallique, montre une complexification progressive des dynamiques culturelles et d'échanges à la transition Bronze-Fer. La quantification et la modélisation de ces phénomènes placent toutefois cette période dans une optique de continuité, qui mènera de fil en aiguille à la mosaïque des cultures et des réseaux d'interactions du premier âge du Fer.

Balzer, Ines, 2009, « Hallstattzeitliche Keramik im Breisgau », dans Bruno Chaume (dir.), La céramique hallstattienne. Approches typologique et chrono-culturelle, Dijon, p. 513-529.

Bolliger, Sabine, 2001, Zürich Wollishofen-Haumeser: spätbronzezeitliche Keramik. Tauchsondierungen 1964-1970, Basel - Egg.

Bonnamour, Louis (dir.), 1990, Du silex à la poudre...4000 ans d'armement en val de Saône, Montagnac.

Bouroche, Jean-Marie \& SAPorta, Gilbert, 1980, L'analyse des données, Paris.

Bradley, Richard, 1990, The passage of arms. An archaeological analysis of prehistoric hoards and votive deposits, Oxford.

CLARKe, David, 1968, Analytical Archaeology, New York.

DeHN, Wolfgang, 1936, « Ein Gräberfeld der älteren Eisenzeit von Laufeld », Trierer Zeitschrift 11, p. 1-49.

DielmanN, Karl, 1952, «Zur Frage "Koberstadter Kultur" », Nassauische Annalen 64, p. ??.

Duindjian, François, 1991, Méthodes pour l'archéologie, Paris.

Dunning, Cynthia \& Rychner, Valentin, 1994, « Archäologische Untersuchungen zum Übergang der Bronze- zur Eisenzeit in der Westschweiz », dans Collectif (éd.), Archäologische Untersuchungen zum Übergang von der Bronze- zur Eisenzeit zwischen Nordsee und Kaukasus (28-30 octobre 1992), Bonn, p. 63-97.

Eggers, Hans-Jürgen, 1950, «Die vergleichende geographisch-kartographische Methode in der Urgeschichtsforschung », Archaeologia Geographica 1, 1, p. 1-3.

Eggers, Hans-Jürgen, 1959, Einführung in die Vorgeschichte, München.

FÉnÉLon, Jean-Pierre, 1981, Qu'est-ce que l'analyse des données ? Exposé accessible aux non-mathématiciens, Paris.

FURIERI, Alessandro (dir.), 2012, «SpatiaLite Manual», dans Gaia-SINS federated projects home-page, http://www.gaia-gis. it/gaia-sins/spatialite-manual-2.3.1.html, page consultée le 28 septembre 2012.

GallaY, Alain, 1986, L'archéologie demain, Paris.

Grimmer-DehN, Beate, 1991, Die Urnenfelderkultur im südöstlichen Oberrheingraben, Stuttgart.

HAFFNer, Alfred, 1973, « Das Grabhügelfeld von Lorentzweiler-Blaschette. Zur Hallstattzeit in Luxemburg », Hémecht 25, p. 401-417.

Hansen, Svend, 1991, Studien zu den Metalldeponierungen während der Urnenfelderzeit im Rhein-Main-Gebiet, Bonn.

HerRmanN, Fritz-Rudolf, 1966, Die Funde der Urnenfelderkultur in Mittel- und Südhessen, Berlin. 
Hornung, Sabine, 2008, Die südöstliche Hunsrück-Eifel-Kultur. Studien zu Späthallstatt- und Frühlatènezeit in der deutschen Mittelgebirgsregion, Bonn.

Jacob-Friesen, Karl-Hermann, 1928, Grundfragen der Urgeschichtsforschung, Stand und Kritik der Forschung über Rassen, Völker und Kulturen in urgeschichtlicher Zeit. Festschrift zur Feier der 75. jährigen Bestehens des Provinzial-Museums, Hannover. JoACHIM, Hans-Eckart, 1968, Die Hunsrück-Eifel-Kultur am Mittelrhein, Köln.

Kiмmig, Wolfgang, 1940, Die Urnenfelderkultur in Baden. Untersucht auf Grund der Gräberfunde, Berlin.

Kimmig, Wolfgang, 1952, « Où en est l'étude de la civilisation des Champs d'Urnes en France, principalement dans l'Est ? », Revue archéologique de l'Est 3, 1 et 3, 3, p. 7-19 et p. 137-172.

Kimmig, Wolfgang, 1954, «Où en est l'étude de la civilisation des Champs d'Urnes en France, principalement dans l'est ? », Revue archéologique de l'Est 5, 1, p. 7-28.

Koenig, Marie-Pierre \& Adam, Anne-Marie, 2005, «La céramique domestique en Alsace de la fin du Bronze final au début de La Tène : essai de synthèse », dans Anne-Marie Adam (dir.), Recherches de Protohistoire alsacienne. La céramique d'habitat du Bronze final III à La Tène ancienne (Supplément à la Revue archéologique de I'Est, 29), Dijon, p. 275-290.

KoEPKE, Hans, 1998, Siedlungs- und Grabfunde der älteren Eisenzeit aus Rheinhessen und dem Gebiet der unteren Nahe, Weissbach.

Kolling, Alfons, 1968, Späte Bronzezeit am Saar und Mosel, Bonn.

Kossack, Georg, 1959, Südbayern während der Hallstattzeit, Berlin.

KRAuSE, Elmar-Björn, 1991, « Brandgräber Typ Laufeld», dans Alfred Haffner \& Andrei Miron (éd.), Studien zur Eisenzeit im Hunsrück-Nahe-Raum, Trier, p. 35-52.

Krausse, Dirk, 2006, Eisenzeitlicher Kulturwandel und Romanisierung im Mosel-Eifel-Raum : die keltisch-römische Siedlung von Wallendorf und ihr archäologisches Umfeld, Mainz.

KuвACH, Wolf, 1994, «Archäologischer Untersuchungen zum Übergang von der Bronze- zur Eisenzeit im südlichen und mittleren Hessen », dans Collectif (éd.), Archäologische Untersuchungen zum Übergang von der Bronze- zur Eisenzeit zwischen Nordsee und Kaukasus (28-30 octobre 1992), Bonn, p. 225-250.

LAbeaune, Régis, 2009, «La céramique hallstattienne dans le Dijonnais: première approche », dans Bruno Chaume (dir.), La céramique hallstattienne, approches typologique et chrono-culturelle, Dijon, p. 181-249.

LebART, Ludovic, Morineau, Alain \& Fénélon, Jean-Pierre, 1982, Traitement des données statistiques. Méthodes et programmes, Paris.

MÄDER, Andreas, 2001, Zürich-Alpenquai I : Die Metallfunde, Zürich - Egg.

MAdSEN, Torsten, 2007, «Multivariate data analysis with PCA, CA and MS », dans ArchaeoInfo, http://www.archaeoinfo.dk/ PDF\%20files/Multivariate\%20data\%20analysis.pdf, page consultée le 1er avril 2013.

MAdSEN, Torsten, 2012, «CA-PCA », dans ArchaeoInfo, http://www.archaeoinfo.dk/CA-PCA/CAPCA\%2022\%2007.zip, page consultée le 28 septembre 2012.

MAISE, Christian, 2001, « Zu Untergliederung der Stufe Ha C/D1 im Breisgau », Fundberichte Baden-Württemberg 25, p. 389-461.

Mentele, Serge, Kuhnle, Gertrud, \& LasserRe, Marina, 2005, « Sainte-Croix-en-Plaine et Houssen « Cora 》 (Haut-Rhin). Contribution à l'étude typo-chronologique de la céramique d'habitat du Hallstatt C», dans Anne-Marie Adam (dir.), Recherches de Protohistoire alsacienne. La céramique d'habitat du Bronze final III à La Tène ancienne (Supplément à la Revue archéologique de I'Est, 23), Dijon, p. 75-142.

Millet, Émilie, 2008, Parures et accessoires vestimentaires : Le costume funéraire dans les régions du Rhin moyen et supérieur, $d u v^{e}$ au III siècle avant J.-C., thèse de doctorat, Université de Bourgogne - Johannes Gutenberg Universität, Mainz.

OLIvier, Laurent, 1993, « Les bracelets rubanés de la Lorraine centrale et les relations entre la Sarre, la Lorraine et la Bourgogne au premier âge du Fer », dans Jean-Michel Massing \& Jean-Paul Petit (éd.), Études offertes à Jean Schaub, BLESA, Metz, p. 345-357.

Pierrevelcin, Gilles, 2012, Les relations entre la Bohême et la Gaule du Ive au i ${ }^{\text {er }}$ siècle avant J. -C. (Dissertationes archaeologicae brunenses / pragensesque, 12) Praha.

Pumain, Denise \& Saint-Julien, Thérèse, 2001, Les interactions spatiales : flux et changements dans l'espace géographique, Paris.

Ramponi, Cécile, 2009, «L'occupation du sol dans l'est lyonnais de la fin de l'âge du Bronze à la fin de l'âge du Fer », dans Marie-Jeanne Roulière-Lambert, Alain Daubigney \& Pierre-Yves Milcent (dir.), De l'âge du Bronze à l'âge du Fer en France et en Europe occidentale, $x^{e}$-VII siècle av. J. -C. La moyenne vallée du Rhône aux âges du Fer (Supplément à la Revue archéologique de I'Est, 27), Dijon, p. 143-164.

ReINHARD, Walter, 2003, Studien zur Hallstatt- und Frühlatènezeit im südöstlichen Saarland, Bliesbruck - Reinheim.

Ruffieux, Mireille, \& Mauvilly, Michel, 2009, «Céramique hallstattienne : données récentes et premier essai de synthèse concernant le canton de Fribourg (Suisse) », dans Bruno Chaume (dir.), La céramique hallstattienne, approches typologique et chrono-culturelle, Dijon, p. 477-511.

Schumacher, Karl, 1918, « Die mittelrheinischen Hallstattkulturen », Germania 2, p. 97-102.

VÉBER, Cécile, 2003, Les dépôts de bronze du Bronze final IIIb en Lorraine, Sarre et Luxembourg : approche technique, Thèse de Doctorat de I'Université de Bourgogne.

VITAL, Joël (dir.), 1993, Habitats et sociétés du Bronze final au premier âge du Fer dans le Jura : les occupations protohistoriques et néolithiques du Pré de la Cour à Montagnieu, Ain, Paris.

VoGt, Emil, 1930, Die spätbronzezeitliche Keramik der Schweiz und ihre Chronologie, Zürich.

VoGT, Emil, 1942, « Der Zierstil der späten Pfahlbaubronzen », Zeitschrift für schweizerische Archäologie und Kunstgeschichte 4, 4, p. 193-206.

ZüRN, Hartwig, 1987, Hallstattzeitliche Grabfunde in Württemberg und Hohenzollern, Stuttgart. 